Received: 10 October 2017

Accepted: 18 December 2017

Published online: 11 January 2018

\section{New anti-inflammatory guaianes from the Atlantic hydrotherm- derived fungus Graphostroma sp. MCCC 3A00421}

\author{
Siwen Niu, Chun-Lan Xie, Jin-Mei Xia, Zhu-Hua Luo, Zongze Shao \& Xian-Wen Yang (1)
}

Nine new guaianes (graphostromanes A-I, 1-9) were isolated from the deep-sea-derived fungus Graphostroma sp. MCCC 3A00421, along with four known ones (10-13). The relative configurations were established mainly by detailed analysis of the NMR and HRESIMS data, while the absolute configurations were assigned using the X-ray crystallography and modified Mosher's method. All isolates were evaluated for their inhibitory effects against lipopolysaccharide (LPS)-induced nitric oxide (NO) production in RAW264.7 macrophages. Graphostromanes F (6) showed remarkable inhibitory effect with an $\mathrm{IC}_{50}$ value of $14.2 \mu \mathrm{M}$, which was even stronger than that of aminoguanidine, a positive control with an $\mathrm{IC}_{50}$ value of $23.4 \mu \mathrm{M}$.

Guaianes are sesquiterpenoids bearing a bicyclo[5.3.0]-decane skeleton with enormously structural diversities, including nor-guaianes ${ }^{1}$, guaiane alkaloids ${ }^{2,3}$, guaiane dimers ${ }^{4,5}$, etc. They possessed a variety of intriguingly biological activities, such as antioxidant ${ }^{2}$, antimalarial ${ }^{6,7}$, antinociceptive ${ }^{7}$, anti-emetic ${ }^{8}$, antitumor ${ }^{9-11}$, anti-inflammatory ${ }^{12,13}$, and antibacteria ${ }^{14,15}$. Guaianes occur mainly in terrestrial plants ${ }^{16}$. Very rarely they were found from terrestrial microorganisms and corals ${ }^{17-19}$. However, they have never been isolated from marine microbes. In our continuing investigation on the deep-sea-derived Graphostroma sp. MCCC 3A0042 $1^{20}$, nine new (1-9) and four known (10-13) guaianes were obtained (Fig. 1). Herein, we report the isolation, structure elucidation, and anti-inflammatory activities of these compounds.

\section{Results and Discussion}

Compound 1 was isolated as a colorless oil. The molecular formula $\mathrm{C}_{15} \mathrm{H}_{26} \mathrm{O}_{2}$ was established on the basis of the $[\mathrm{M}+\mathrm{H}]^{+}$ionic peak at $\mathrm{m} / z 239.2044$ in its positive HRESIMS, requiring three indices of hydrogen deficiency. The ${ }^{1} \mathrm{H}$ NMR spectroscopic data (Table 1$)$ showed two singlet $\left(\delta_{\mathrm{H}} 1.40\right.$ and 1.76$)$ and one doublet $\left(\delta_{\mathrm{H}} 1.08\right)$ methyls, one exomethylene $\left(\delta_{\mathrm{H}} 4.83,4.72\right)$, and one oxygenated methine $\left(\delta_{\mathrm{H}} 4.22\right)$. These signals were resonance in the ${ }^{13} \mathrm{C}$ NMR data (Table 2$)$ as three methyls $\left(\delta_{\mathrm{C}} 14.4,19.9\right.$, and 29.4$)$, one exocyclic methylene $\left(\delta_{\mathrm{C}} 107.8\right)$, one oxygenated methine $\left(\delta_{\mathrm{C}} 77.4\right)$. Altogether, the ${ }^{1} \mathrm{H}$ and ${ }^{13} \mathrm{C}$ NMR exhibited 15 carbons attributing to three methyls, five methylenes, five methines, and two nonprotonated carbons (one $s p^{2}$ at $\delta_{\mathrm{C}} 152.7$ and one oxygenated $s p^{3}$ at $\delta_{\mathrm{C}}$ 73.0). Since an olefinic bond accounted for one unsaturation degree, a bicyclic framework was required for 1 . In the COSY spectrum, one spin coupling system from $\mathrm{H}-1$ through $\mathrm{H}_{2}-2$ to $\mathrm{H}-3 / \mathrm{H}-4 / \mathrm{H}-5 / \mathrm{H}_{2}-6 / \mathrm{H}-7 / \mathrm{H}_{2}-8 / \mathrm{H}_{2}-$ 9, from $\mathrm{H}-5$ to $\mathrm{H}-1$, and from $\mathrm{H}-4$ to $\mathrm{H}_{3}-15$ constructed a long fragment. In the HMBC spectrum, correlations from 14-Me $\left(\delta_{\mathrm{H}} 1.40\right)$ to $\mathrm{C}-1\left(\delta_{\mathrm{C}} 53.0\right), \mathrm{C}-9\left(\delta_{\mathrm{C}} 37.8\right)$, and C-10 $\left(\delta_{\mathrm{C}} 73.0\right)$ and from 12-Me to C-7, C-11 $\left(\delta_{\mathrm{C}} 152.7\right)$, and $\mathrm{C}-13\left(\delta_{\mathrm{C}} 107.8\right)$ established 1 as a guaiane sesquiterpene (Fig. 2$)$.

In the NOESY spectrum, correlations from $\mathrm{H}-5\left(\delta_{\mathrm{H}} 2.66\right)$ to $\mathrm{H}-1 / \mathrm{H}-4 / \mathrm{H}-7\left(\delta_{\mathrm{H}} 2.59\right)$ and from Me-15 to Me-14 and H-3 disclosed that H-1, H-4, H-5, and $\mathrm{H}-7$ were on the same face, whereas $\mathrm{H}-3,14-\mathrm{Me}$, and $15-\mathrm{Me}$ were on the opposite side (Fig. 2). This was unambiguously confirmed by the $\mathrm{Cu}-\mathrm{K} \alpha \mathrm{X}$-ray crystallography (Fig. 3). Therefore, 1 was determined to be $(1 R, 3 R, 4 R, 5 S, 7 R, 10 R)-11(13)$-en-3,10-dihydroxyguaiene, and named graphostromane A.

State Key Laboratory Breeding Base of Marine Genetic Resources, Key Laboratory of Marine Genetic Resources, Fujian Key Laboratory of Marine Genetic Resources, South China Sea Bio-Resource Exploitation and Utilization Collaborative Innovation Center, Third Institute of Oceanography, State Oceanic Administration, 184 Daxue Road, Xiamen, 361005, People's Republic of China. Correspondence and requests for materials should be addressed to X.W.Y. (email: yangxianwen@tio.org.cn) 


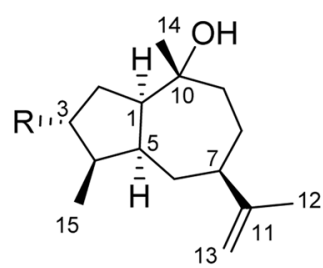

1. $\mathrm{R}=\mathrm{OH}$

2. $\mathrm{R}=\mathrm{H}$<smiles>C[C@]1(O)CC[C@@H]2[C@H](O)CC[C@@H]([C@@](C)(O)CO)C[C@H]21</smiles>

8<smiles>[R]C1C[C@@H]2[C@@H](C[C@@H](C(=C)C)CC[C@@H](C)[C@@H]2C)[C@H]1C</smiles>

3. $\mathrm{R}=\mathrm{OH}$

4. $\mathrm{R}=\mathrm{O}$<smiles>C[C@H](C(=O)O)[C@H]1CC[C@@H](O)[C@H]2CC[C@@H](C)[C@H]2C1</smiles>

9<smiles>[R]CC(=C)[C@@H]1CC[C@H](O)[C@H]2[C@@H](C)C[C@@H](O)[C@H]2C1</smiles>

5. $\mathrm{R}=\mathrm{H}$

6. $\mathrm{R}=\mathrm{OH}$<smiles>[R]C1C[C@H]2[C@@H](C)[C@@H](C)CC[C@](C)(O)[C@@H]2C1</smiles>

11. $\mathrm{R}=\mathrm{H}$

12. $\mathrm{R}=\mathrm{OH}$<smiles>[R]OC[C@](C)(O)[C@@H]1CC[C@@](C)(O)[C@@H]2[C@@H](C)C[C@@H](O)[C@@H]2C1</smiles>

7. $R=A c$

10. $R=H$<smiles>C[C@H]1C[C@H](C(C)(O)CO)C[C@@H](O)[C@]2(C)O[C@H]1CC[C@@H]2C</smiles>

13

Figure 1. Chemical structures of 1-13.

Compound 2 showed the formula molecular of $\mathrm{C}_{15} \mathrm{H}_{26} \mathrm{O}$ as established by the positive HRESIMS at $\mathrm{m} / z$ 223.2057 $[\mathrm{M}+\mathrm{H}]^{+}$. The ${ }^{1} \mathrm{H}$ and ${ }^{13} \mathrm{C}$ NMR spectra were nearly identical to those of 1 except that a methylene $\left(\delta_{\mathrm{C}}\right.$ 32.1) instead of an oxygenated methine was located at C-3 position. This observation was evidenced by the HMBC correlation of 15-Me $\left(\delta_{\mathrm{H}} 0.92\right)$ to the methylene unit. On the basis of its key NOESY correlations (Fig. 4) and the similar optical rotation value $\left([\alpha]_{\mathrm{D}}^{25}-28.5\right.$ for 2 , while -22.0 for $\left.\mathbf{1}\right), 2$ was therefore deduced to be $(1 R, 4 S, 5 S, 7 R, 10 R)-11(13)$-en-10-hydroxyguaiene, and named graphostromane $\mathrm{B}$.

Compound 3 showed the same molecule formula as that of 1 by the positive HRESIMS at $\mathrm{m} / z 261.1829$ (calcd for $\mathrm{C}_{15} \mathrm{H}_{26} \mathrm{O}_{2} \mathrm{Na}, 261.1830$ ). Interestingly, it also exhibited almost the same ${ }^{1} \mathrm{H}$ and ${ }^{13} \mathrm{C}$ NMR spectra, except for the shielded chemical shift of $\mathrm{H}-3\left(\delta_{\mathrm{H}} 3.87\right)$ and its peak pattern, indicating 3 and $\mathbf{1}$ might be C-3 stereoisomers. The NOESY correlations between $\mathrm{H}-3 / \mathrm{H}-1\left(\delta_{\mathrm{H}} 2.61\right), \mathrm{H}-1 / \mathrm{H}-9 \mathrm{a}\left(\delta_{\mathrm{H}} 1.98\right), \mathrm{H}-9 \mathrm{a} / \mathrm{H}-7$, and $\mathrm{H}-9 \mathrm{~b}\left(\delta_{\mathrm{H}} 2.24\right)$ to $\mathrm{H}_{3}$ $14\left(\delta_{\mathrm{H}} 1.50\right)$ deduced $\mathrm{H}-1, \mathrm{H}-3$, and $\mathrm{H}-7$ were in the $\alpha$ orientations, while $\mathrm{H}_{3}-14$ was $\beta$-oriented. However, it is difficult to establish the relative configurations of C- 4 and C- 5 positions by the NOESY correlations because of the overlap signals of $\mathrm{H}-4\left(\delta_{\mathrm{H}} 1.67\right)$ and $\mathrm{H}-5\left(\delta_{\mathrm{H}} 1.69\right)$. Therefore, all four possible stereoisomers including a pair of cis-fused C-4 epimers [ $\left(1 R^{*}, 3 S^{*}, 4 R^{*}, 5 S^{*}, 7 R^{*}, 10 R^{*}\right)-3 \mathbf{a}$ and $\left.\left(1 R^{*}, 3 S^{*}, 4 S^{*}, 5 S^{*}, 7 R^{*}, 10 R^{*}\right)-3 \mathbf{b}\right]$ and a pair of trans-fused C-4 epimers $\left[\left(1 R^{*}, 3 S^{*}, 4 R^{*}, 5 R^{*}, 7 R^{*}, 10 R^{*}\right)-3 \mathrm{c}\right.$ and $\left.\left(1 R^{*}, 3 S^{*}, 4 S^{*}, 5 R^{*}, 7 R^{*}, 10 R^{*}\right)-3 \mathrm{~d}\right]$ were subjected for the theoretical calculation of the CMR data at mPW1PW91/6-311+G(2d,p) level using the IEFPCM model in pyridine- $d_{5}$ by Gaussian 09 . As shown in Fig. 5 , $3 \mathrm{c}$ displayed the smallest deviation, suggesting the relative configuration of 3 to be $1 R^{*}, 3 S^{*}, 4 R^{*}, 5 R^{*}, 7 R^{*}$, and $10 R^{*}$. By the modified Mosher's method, C-3 was determined to be $S$ configuration (Fig. 6$)^{21}$. Based on the above evidences, 3 was then established to be $(1 R, 3 S, 4 R, 5 R, 7 R, 10 R)$ 11(13)-en-3,10-dihydroxyguaiene, and named graphostromane $\mathrm{C}$.

Compound 4 was established the molecular formula $\mathrm{C}_{15} \mathrm{H}_{24} \mathrm{O}_{2}$ on the basis of its HRESIMS. The ${ }^{1} \mathrm{H}$ and ${ }^{13} \mathrm{C}$ NMR spectra were very similar to those of 3 except that a ketone group $\left(\delta_{\mathrm{C}} 220.6\right)$ rather than a hydroxy unit was located at C-3. The assumption was corroborated by the HMBC relationships from $15-\mathrm{Me}\left(\delta_{\mathrm{H}} 1.08\right)$ to the carbonyl carbon. Therefore, 4 was assigned as $(1 R, 4 S, 5 S, 7 R, 10 R)$-3-oxo-11(13)-en-10-hydroxyguaiene, and named graphostromane D.

Compound 5 exhibited the same molecular formula as that of $\mathbf{1}$ according to its positive HRESIMS at $\mathrm{m} / \mathrm{z}$ $261.1833[\mathrm{M}+\mathrm{Na}]^{+}$. Close comparison of its ${ }^{1} \mathrm{H}$ and ${ }^{13} \mathrm{C}$ NMR spectra to those of 1 revealed a general similarity except that the hydroxy unit should be attached to C-2 instead of C-3 in 5. This was confirmed by the HMBC correlations of $15-\mathrm{Me}$ to the methylene unit at $\delta_{\mathrm{C}} 43.1$ of the C-3 position. In the NOESY spectrum, cross-peaks from $\mathrm{H}-9 \mathrm{a}\left(\delta_{\mathrm{H}} 1.85\right)$ to Me-14 $\left(\delta_{\mathrm{H}} 1.33\right)$ and $\mathrm{H}-2$ revealed $\mathrm{H}-2$ was in $\beta$ orientation (Fig. 4$)$. By the modified Mosher's method, C-2 was determined as $S$-configuration (Fig. 6). Accordingly, 5 was established to be $(1 S, 2 S, 4 S, 5 S, 7 R, 10 R)-11(13)$-en-2,10-dihydroxyguaiene, and named graphostromane $\mathrm{E}$.

Compound 6 was assigned the molecular formula $\mathrm{C}_{15} \mathrm{H}_{26} \mathrm{O}_{3}$ by the HRESIMS at $m / z 277.1767[\mathrm{M}+\mathrm{Na}]^{+}$. The ${ }^{1} \mathrm{H}$ and ${ }^{13} \mathrm{C}$ NMR spectra were nearly identical to those of $\mathbf{5}$, except for an additional hydroxy unit at $\mathrm{C}-12\left(\delta_{\mathrm{C}} 64.1\right)$. The assumption was evidenced by the $\mathrm{HMBC}$ correlations from $\mathrm{H}_{2}-13\left(\delta_{\mathrm{H}} 5.47,5.08\right)$ to C-7 $\left(\delta_{\mathrm{C}} 43.7\right), \mathrm{C}-11\left(\delta_{\mathrm{C}} 157.9\right)$, and C-12. Accordingly, 6 was determined to be $(1 S, 2 S, 4 S, 5 S, 7 R, 10 R)-11(13)$-en2,10,12-trihydroxyguaiene, and named graphostromane $\mathrm{F}$.

Compound 7 gave a molecular formula $\mathrm{C}_{17} \mathrm{H}_{30} \mathrm{O}_{5}$ from its positive HRESIMS at $m / z 337.1979[\mathrm{M}+\mathrm{Na}]^{+}$. Its ${ }^{1} \mathrm{H}$ and ${ }^{13} \mathrm{C}$ NMR spectra were close to those of $(1 S, 2 S, 4 S, 5 S, 7 R, 10 R)$-guaiane-2,10,11,12-tetraol (10) ${ }^{22}$, except for an additional acetyl group $\left(\delta_{\mathrm{H}} 1.99 ; \delta_{\mathrm{C}} 20.6,170.7\right)$ at $\mathrm{C}-12$. This was evidenced by the HMBC cross-peaks from $\mathrm{H}_{2}-12\left(\delta_{\mathrm{H}} 4.42\right)$ to the carbonyl $\left(\delta_{\mathrm{C}} 170.7\right)$ of the acetyl group. The absolute configuration of $\mathrm{C}-2$ was established to be $S$ on the basis of the modified Mosher's method (Fig. 6), which was further corroborated by the X-ray 


\begin{tabular}{|c|c|c|c|c|c|c|c|c|c|}
\hline no. & $1^{\mathrm{a}}$ & $2^{\mathrm{b}}$ & $3^{a}$ & $4^{a}$ & $5^{b}$ & $6^{\mathrm{a}}$ & $7^{\mathrm{a}}$ & $8^{\mathrm{a}}$ & $9^{b}$ \\
\hline 1 & 2.93, dt $(8.3,9.9)$ & $2.17, \mathrm{~m}$ & $2.61, \mathrm{~m}$ & $2.71, \mathrm{~m}$ & $\begin{array}{l}2.07, \text { dd }(9.9, \\
7.6)\end{array}$ & $2.51, \mathrm{~m}$ & $2.55, \mathrm{~m}$ & $3.20, \mathrm{~m}$ & $2.12, \mathrm{~m}$ \\
\hline 2 & $2.22, \mathrm{~m}$ & $1.73, \mathrm{~m} ; 1.58, \mathrm{~m}$ & $2.67, \mathrm{~m} ; 2.26, \mathrm{~m}$ & $\begin{array}{l}3.18, \text { ddd }(19.7,4.2, \\
1.3) ; 2.51, \text { dd }(19.4,9.7)\end{array}$ & $4.27, \mathrm{q}(7.2)$ & $4.70, \mathrm{q}(7.3)$ & $4.64, \mathrm{q}(6.9)$ & $2.10, \mathrm{~m} ; 1.76, \mathrm{~m}$ & $\begin{array}{l}1.72, \mathrm{~m} \\
1.54, \mathrm{~m}\end{array}$ \\
\hline 3 & $4.22, \mathrm{dt}(9.5,3.4)$ & $1.73, \mathrm{~m} ; 1.28, \mathrm{~m}$ & $3.87, \mathrm{dt}(6.8,9.1)$ & & $1.70, \mathrm{~m}$ & $1.96, \mathrm{~m} ; 1.91, \mathrm{~m}$ & $1.95, \mathrm{~m}$ & $\begin{array}{l}2.04, \mathrm{~m} ; 1.92, \mathrm{td} \\
(12.8,3.7)\end{array}$ & $\begin{array}{l}1.72, \mathrm{~m} \\
1.25, \mathrm{~m}\end{array}$ \\
\hline 4 & $2.29, \mathrm{~m}$ & $2.03, \mathrm{~m}$ & $1.67, \mathrm{~m}$ & $2.04, \mathrm{~m}$ & $2.21, \mathrm{~m}$ & $2.15, \mathrm{~m}$ & $2.28, \mathrm{~m}$ & & $2.02, \mathrm{~m}$ \\
\hline 5 & $2.66, \mathrm{~m}$ & $2.04, \mathrm{~m}$ & $1.69, \mathrm{~m}$ & $2.04, \mathrm{~m}$ & $2.38, \mathrm{~m}$ & $2.51, \mathrm{~m}$ & $2.53, \mathrm{~m}$ & $2.54, \mathrm{~m}$ & $1.96, \mathrm{~m}$ \\
\hline 6 & $1.63, \mathrm{~m} ; 1.47, \mathrm{td}(13.3,2.8)$ & $1.40, \mathrm{~m} ; 1.27, \mathrm{~m}$ & $\begin{array}{l}\text { 1.84, br d (12.9); } 1.57, \text { br } \\
\mathrm{t}(11.5)\end{array}$ & $1.99, \mathrm{~m} ; 1.48, \mathrm{~m}$ & $1.42, \mathrm{~m} ; 1.38, \mathrm{~m}$ & $1.64, \mathrm{~m}$ & $\begin{array}{l}2.22, \mathrm{~m} \\
1.29, \mathrm{~m}\end{array}$ & $\begin{array}{l}\text { 2.63, dd (13.5, } \\
3.2) ; 1.36, \mathrm{~m}\end{array}$ & $\begin{array}{l}1.44, \mathrm{~m} \\
1.08, \mathrm{~m}\end{array}$ \\
\hline 7 & $2.59, \operatorname{td}(10.3,4.9)$ & $2.34, \mathrm{~m}$ & 2.10, brt $(11.0)$ & $2.29, \operatorname{td}(10.9,2.2)$ & $2.19, \mathrm{~m}$ & $2.39, \mathrm{~m}$ & $2.27, \mathrm{~m}$ & $2.51, \mathrm{~m}$ & $2.05, \mathrm{~m}$ \\
\hline 8 & $2.09, \mathrm{~m} ; 1.57, \mathrm{~m}$ & $1.87, \mathrm{~m} ; 1.43, \mathrm{~m}$ & $1.76, \mathrm{~m} ; 1.64, \mathrm{~m}$ & $1.87, \mathrm{~m} ; 1.54, \mathrm{~m}$ & $1.75, \mathrm{~m} ; 1.47, \mathrm{~m}$ & $1.98, \mathrm{~m} ; 1.68, \mathrm{~m}$ & \begin{tabular}{|l|}
$2.16, \mathrm{~m}$ \\
$1.43, \mathrm{~m}$ \\
\end{tabular} & $2.28, \mathrm{~m} ; 1.49, \mathrm{~m}$ & $\begin{array}{l}1.87, \mathrm{~m} \\
1.35, \mathrm{~m}\end{array}$ \\
\hline 9 & $2.11, \mathrm{~m} ; 1.80, \mathrm{~m}$ & $1.91, \mathrm{~m} ; 1.55, \mathrm{~m}$ & $2.24, \mathrm{~m} ; 1.98, \operatorname{td}(13.5,3.2)$ & $2.19, \mathrm{~m} ; 1.91, \mathrm{~m}$ & $1.85, \mathrm{~m} ; 1.69, \mathrm{~m}$ & $2.15, \mathrm{~m} ; 1.95, \mathrm{~m}$ & $\begin{array}{l}2.17, \mathrm{~m} \\
1.91, \mathrm{~m}\end{array}$ & $2.21, \mathrm{~m} ; 1.83, \mathrm{~m}$ & $\begin{array}{l}1.90, \mathrm{~m} \\
1.51, \mathrm{~m}\end{array}$ \\
\hline 11 & & & & & & & & & $2.30, \mathrm{~m}$ \\
\hline 12 & $1.76, \mathrm{~s}$ & $1.70, \mathrm{~s}$ & $1.76, \mathrm{~s}$ & $1.75, \mathrm{~s}$ & $1.70, \mathrm{~s}$ & $4.47, \mathrm{t}(1.4)$ & $4.42, \mathrm{~s}$ & $\begin{array}{l}3.99, \mathrm{~d}(10.5) ; 3.89, \\
\mathrm{~d}(10.5)\end{array}$ & \\
\hline 13 & $4.83, \mathrm{~d}(1.7) ; 4.72, \mathrm{~m}$ & $4.66, \mathrm{~m} ; 4.58, \mathrm{~m}$ & $4.84, \mathrm{~d}(1.5) ; 4.75, \mathrm{~m}$ & $4.83, \mathrm{~m} ; 4.74, \mathrm{~m}$ & $4.65, \mathrm{~m} ; 4.59, \mathrm{~m}$ & $5.47, \mathrm{q}(1.8) ; 5.08, \mathrm{~m}$ & $1.36, \mathrm{~s}$ & $1.41, \mathrm{~s}$ & $\begin{array}{l}1.10, \mathrm{~d} \\
(7.0)\end{array}$ \\
\hline 14 & $1.40, \mathrm{~s}$ & $1.18, \mathrm{~s}$ & $1.50, \mathrm{~s}$ & $1.30, \mathrm{~s}$ & $1.33, \mathrm{~s}$ & $1.61, \mathrm{~s}$ & $1.68, \mathrm{~s}$ & $1.46, \mathrm{~s}$ & $1.15, \mathrm{~s}$ \\
\hline 15 & $1.08, \mathrm{~d}(7.2)$ & $0.92, \mathrm{~d}(6.8)$ & $1.20, \mathrm{~d}(6.0)$ & $1.08, \mathrm{~d}(6.4)$ & $0.89, \mathrm{~d}(7.2)$ & $0.83, \mathrm{~d}(7.3)$ & $0.93, \mathrm{~d}(7.3)$ & $1.54, \mathrm{~s}$ & $\begin{array}{l}0.92, \mathrm{~d} \\
(7.0)\end{array}$ \\
\hline Ac & & & & & & & $1.99, \mathrm{~s}$ & & \\
\hline $2-\mathrm{OH}$ & & & & & & & 5.75 , br s & & \\
\hline $10-\mathrm{OH}$ & & & & 5.78, br s & & & 5.63 , br s & & \\
\hline $11-\mathrm{OH}$ & & & & & & & 5.96 , br s & & \\
\hline
\end{tabular}

Table 1. ${ }^{1} \mathrm{H}$ NMR spectroscopic data of $\mathbf{1 - 9}$ recorded at $400 \mathrm{MHz}$ ( $\delta$ in ppm, $J$ in $\mathrm{Hz}$ within the parenthesis). ${ }^{\mathrm{a}}$ Measured in pyridine- $d_{5} .{ }^{\mathrm{b}}$ Measured in $\mathrm{CD}_{3} \mathrm{OD}$.

single-crystal experiment (Fig. 7). Therefore, 7 was unambiguously determined to be $(1 S, 2 S, 4 S, 5 S, 7 R, 10 R, 11 R)$ 12-acetyl-2,10,11-trihydroxyguaiane, and named graphostromane $\mathrm{G}$.

Compound 8 had the molecular formula of $\mathrm{C}_{15} \mathrm{H}_{28} \mathrm{O}_{4}$ as established by its HRESIMS. Analysis of the 1D and 2D NMR spectra established the structure of $\mathbf{8}$ was closely related to 10, except that the hydroxy group was located at C-4 instead of C-2. This was evidenced by the HMBC correlations from $15-\mathrm{Me}\left(\delta_{\mathrm{H}} 1.54\right)$ to C-3 $\left(\delta_{\mathrm{C}} 39.6\right), \mathrm{C}-4$ $\left(\delta_{\mathrm{C}} 80.9\right)$, and C-5 $\left(\delta_{\mathrm{C}} 54.5\right)$. The NOE cross-peaks from H-2a $\left(\delta_{\mathrm{H}} 2.10\right)$ to 14-Me $\left(\delta_{\mathrm{H}} 1.46\right)$ and 15-Me revealed that 4 -OH was in $\alpha$-oriented. Therefore, 8 was identified to be $(1 R, 4 R, 5 R, 7 R, 10 R)-4,10,11$-trihydroxyguaiane, and named graphostromane $\mathrm{H}$.

Compound 9 was assigned the molecular formula $\mathrm{C}_{15} \mathrm{H}_{26} \mathrm{O}_{3}$ on the basis of the HRESIMS at $m / z 277.1783$ $[\mathrm{M}+\mathrm{Na}]^{+}$. Its ${ }^{1} \mathrm{H}$ and ${ }^{13} \mathrm{C} \mathrm{NMR}$ spectra were very similar to those of $(1 R, 4 S, 5 S, 7 R, 10 R, 11 S)$-guaiane10,11,12-triol (11 $)^{15}$ except that a hydroxy group was absent at C-11, while a carboxyl moiety $\left(\delta_{\mathrm{C}} 180.2\right)$ instead of an oxymethylene was attached to $\mathrm{C}-11$. This was corroborated by the HMBC correlations form 13-Me $\left(\delta_{\mathrm{H}} 1.10\right)$ to $\mathrm{C}-7\left(\delta_{\mathrm{C}} 41.4\right), \mathrm{C}-11\left(\delta_{\mathrm{C}} 47.0\right)$, and $\mathrm{C}-12\left(\delta_{\mathrm{C}} 180.2\right)$. By the theoretical calculation of CMR spectrum, C-11 was assigned as $R$-configuration (Fig. 8). Therefore, 9 was established to be $(1 R, 4 S, 5 S, 7 R, 10 R, 11 R)$-10-hydroxyguai12-oic acid, and named graphostromane I.

By comparison of the NMR, MS, and OR data with those published in the literature, four known guaianes were identified as $(1 S, 2 S, 4 S, 5 S, 7 R, 10 R)$-guaiane-2,10,11,12-tetraol $(\mathbf{1 0})^{22},(1 R, 4 S, 5 S, 7 R, 10 R, 11 S)$-guaiane$10,11,12$-triol $(11)^{15},(1 R, 3 S, 4 R, 5 S, 7 R, 10 R, 11 S)$-guaiane-3,10,11,12-tetraol (12) ${ }^{15}$, and $(1 R, 4 S, 5 S, 7 S, 9 R, 10 S, 11 R)$-guaiane-9,10,11,12-tetraol $(13)^{15}$

All isolates were evaluated for their anti-inflammatory activities against LPS-induced NO production in RAW264.7 macrophages (Table 3). Compound 6 exhibited remarkable anti-inflammatory activity with an IC 50 value of $14.2 \mu \mathrm{M}$, which was stronger than that of the positive control, aminoguanidine, with an $\mathrm{IC}_{50}$ of $23.4 \mu \mathrm{M}$. In addition, 4, 9, and 13 showed weak anti-inflammatory activities with $\mathrm{IC}_{50}$ values of 72.9, 79.1, and 88.2 $\mu \mathrm{M}$, respectively.

In conclusion, chemical investigation on the deep-sea-derived fungus Graphostroma sp. MCCC 3 A00421 led to the isolation of 9 new (graphostromanes A-I, 1-9) and 4 known (10-13) guaianes. They are first examples of guaiane sesquiterpenoids reported from the marine-derived fungi. Additionally, $\mathbf{6}$ showed potent anti-inflammatory activity against LPS-induced NO production in RAW264.7 macrophages, indicating its potential usage for anti-inflammatory drugs.

\section{Materials and Methods}

General Experimental Procedures. An automatic polarimeter Rudolph IV Autopol was used for recording optical rotation data at $25^{\circ} \mathrm{C}$. A Xevo G2 Q-TOF mass spectrometer was used for measuring HRESIMS. A Bruker Avance $400 \mathrm{MHz}$ NMR spectrometer was used for measuring ${ }^{1} \mathrm{H},{ }^{13} \mathrm{C}, \mathrm{HSQC}, \mathrm{COSY}, \mathrm{HMBC}$, and NOESY spectra. Chemical shifts $(\delta)$ were expressed in ppm referring to the solvent peaks, and coupling constants are in Hz. A Bruker D8 Advance X-ray single-crystal diffractometer was used for measuring X-ray data with $\mathrm{Cu} \mathrm{K \alpha}$ radiation. Column chromatography (CC) were performed on Sephadex LH-20 (18-110 $\mu$ m, Pharmacia, Uppsala, Sweden), silica gel (100-200 or 200-300 mush, Qingdao Marine Chemistry Co. Ltd, Qingdao, China), and ODS 


\begin{tabular}{|c|c|c|c|c|c|c|c|c|c|}
\hline no. & $1^{\mathrm{a}}$ & $2^{b}$ & $3^{a}$ & $4^{\mathrm{a}}$ & $5^{\mathrm{b}}$ & $6^{a}$ & $7^{\mathrm{a}}$ & $8^{a}$ & $9^{b}$ \\
\hline 1 & $53.0, \mathrm{CH}$ & $56.0, \mathrm{CH}$ & 49.6, $\mathrm{CH}$ & $47.3, \mathrm{CH}$ & $62.7, \mathrm{CH}$ & $61.2, \mathrm{CH}$ & 62.6, $\mathrm{CH}$ & $52.7, \mathrm{CH}$ & $56.1, \mathrm{CH}$ \\
\hline 2 & $36.8, \mathrm{CH}_{2}$ & 27.1, $\mathrm{CH}_{2}$ & $37.4, \mathrm{CH}_{2}$ & $40.2, \mathrm{CH}_{2}$ & $75.2, \mathrm{CH}$ & $74.1, \mathrm{CH}$ & 74.0, $\mathrm{CH}$ & $25.9, \mathrm{CH}_{2}$ & $27.0, \mathrm{CH}_{2}$ \\
\hline 3 & $77.4, \mathrm{CH}$ & $32.1, \mathrm{CH}_{2}$ & $77.7, \mathrm{CH}$ & 220.6, C & $43.1, \mathrm{CH}_{2}$ & $42.5, \mathrm{CH}_{2}$ & $43.0, \mathrm{CH}_{2}$ & $39.6, \mathrm{CH}_{2}$ & $31.8, \mathrm{CH}_{2}$ \\
\hline 4 & $48.0, \mathrm{CH}$ & $40.1, \mathrm{CH}$ & $49.0, \mathrm{CH}$ & $49.7, \mathrm{CH}$ & $37.0, \mathrm{CH}$ & $35.8, \mathrm{CH}$ & $36.3, \mathrm{CH}$ & $80.9, \mathrm{C}$ & $40.3, \mathrm{CH}$ \\
\hline 5 & $44.9, \mathrm{CH}$ & $47.0, \mathrm{CH}$ & $46.0, \mathrm{CH}$ & $45.5, \mathrm{CH}$ & $46.2, \mathrm{CH}$ & $44.4, \mathrm{CH}$ & $46.0, \mathrm{CH}$ & $54.5, \mathrm{CH}$ & $47.3, \mathrm{CH}$ \\
\hline 6 & $30.4, \mathrm{CH}_{2}$ & 29.6, $\mathrm{CH}_{2}$ & $37.0, \mathrm{CH}_{2}$ & $38.3, \mathrm{CH}_{2}$ & $33.0, \mathrm{CH}_{2}$ & $34.0, \mathrm{CH}_{2}$ & $26.2, \mathrm{CH}_{2}$ & $26.0, \mathrm{CH}_{2}$ & $26.1, \mathrm{CH}_{2}$ \\
\hline 7 & $46.7, \mathrm{CH}$ & $47.1, \mathrm{CH}$ & $47.9, \mathrm{CH}$ & $44.9, \mathrm{CH}$ & $48.6, \mathrm{CH}$ & $43.7, \mathrm{CH}$ & $46.4, \mathrm{CH}$ & $45.5, \mathrm{CH}$ & $41.4, \mathrm{CH}$ \\
\hline 8 & $29.7, \mathrm{CH}_{2}$ & $29.7, \mathrm{CH}_{2}$ & $32.0, \mathrm{CH}_{2}$ & $32.5, \mathrm{CH}_{2}$ & $31.1, \mathrm{CH}_{2}$ & 31.6, $\mathrm{CH}_{2}$ & $26.2, \mathrm{CH}_{2}$ & $25.9, \mathrm{CH}_{2}$ & $28.0, \mathrm{CH}_{2}$ \\
\hline 9 & $37.8, \mathrm{CH}_{2}$ & $36.8, \mathrm{CH}_{2}$ & $46.2, \mathrm{CH}_{2}$ & $45.0, \mathrm{CH}_{2}$ & $42.0, \mathrm{CH}_{2}$ & $43.8, \mathrm{CH}_{2}$ & $40.7, \mathrm{CH}_{2}$ & $38.0, \mathrm{CH}_{2}$ & $35.4, \mathrm{CH}_{2}$ \\
\hline 10 & 73.0, C & $75.2, \mathrm{C}$ & 73.9, C & 73.3, C & 75.7, C & $74.3, \mathrm{C}$ & $73.9, \mathrm{C}$ & $73.4, \mathrm{C}$ & $75.3, \mathrm{C}$ \\
\hline 11 & 152.7, C & 153.4, C & $152.5, \mathrm{C}$ & 152.1, C & 153.5, C & 157.9, C & $73.4, \mathrm{C}$ & $75.3, \mathrm{C}$ & $47.0, \mathrm{CH}$ \\
\hline 12 & $19.9, \mathrm{CH}_{3}$ & $20.3, \mathrm{CH}_{3}$ & $20.1, \mathrm{CH}_{3}$ & $19.9, \mathrm{CH}_{3}$ & $20.3, \mathrm{CH}_{3}$ & 64.1, $\mathrm{CH}_{2}$ & $70.6, \mathrm{CH}_{2}$ & $68.8, \mathrm{CH}_{2}$ & $180.2, \mathrm{C}$ \\
\hline 13 & $107.8, \mathrm{CH}_{2}$ & 108.4, $\mathrm{CH}_{2}$ & $107.8, \mathrm{CH}_{2}$ & 108.0, $\mathrm{CH}_{2}$ & 108.4, $\mathrm{CH}_{2}$ & $105.8, \mathrm{CH}_{2}$ & $20.3, \mathrm{CH}_{3}$ & $19.7, \mathrm{CH}_{3}$ & $13.5, \mathrm{CH}_{3}$ \\
\hline 14 & $29.4, \mathrm{CH}_{3}$ & $30.1, \mathrm{CH}_{3}$ & $24.1, \mathrm{CH}_{3}$ & $24.4, \mathrm{CH}_{3}$ & $28.0, \mathrm{CH}_{3}$ & $26.9, \mathrm{CH}_{3}$ & $29.1, \mathrm{CH}_{3}$ & $29.6, \mathrm{CH}_{3}$ & $30.5, \mathrm{CH}_{3}$ \\
\hline 15 & $14.4, \mathrm{CH}_{3}$ & $16.8, \mathrm{CH}_{3}$ & $16.4, \mathrm{CH}_{3}$ & $13.9, \mathrm{CH}_{3}$ & $16.8, \mathrm{CH}_{3}$ & $16.7, \mathrm{CH}_{3}$ & $16.6, \mathrm{CH}_{3}$ & $25.0, \mathrm{CH}_{3}$ & $16.6, \mathrm{CH}_{3}$ \\
\hline $\mathrm{Ac}$ & & & & & & & $20.6, \mathrm{CH}_{3} 170.7, \mathrm{C}$ & & \\
\hline
\end{tabular}

Table 2. ${ }^{13} \mathrm{C}$ NMR spectroscopic data of compounds 1-9. ${ }^{\mathrm{a}}$ Measured in pyridine- $d_{5}$ at $100 \mathrm{MHz} .{ }^{\mathrm{b}} \mathrm{Measured}$ in $\mathrm{CD}_{3} \mathrm{OD}$ at $100 \mathrm{MHz}$.
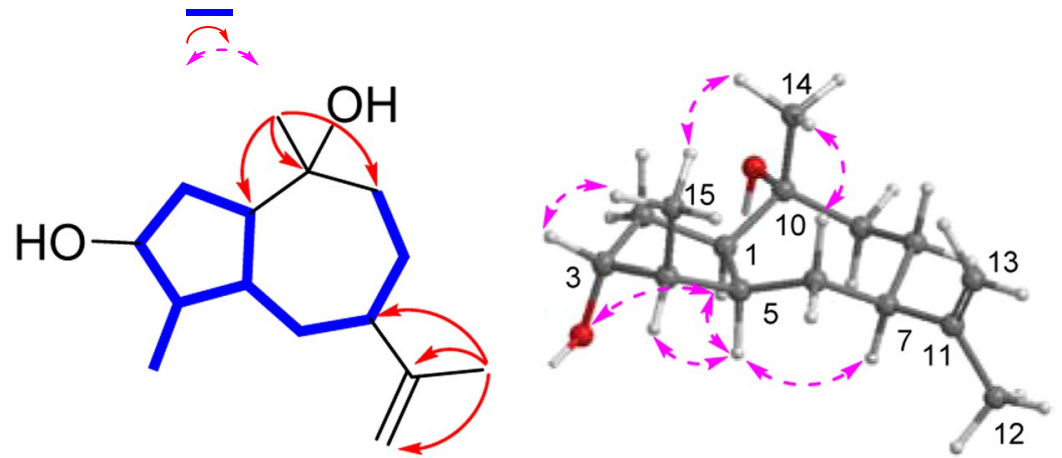

Figure 2. Selected COSY $(-), \operatorname{HMBC}(\curvearrowright)$, and NOESY $\left(\bullet^{-} \vee\right)$ correlations of $\mathbf{1}$.

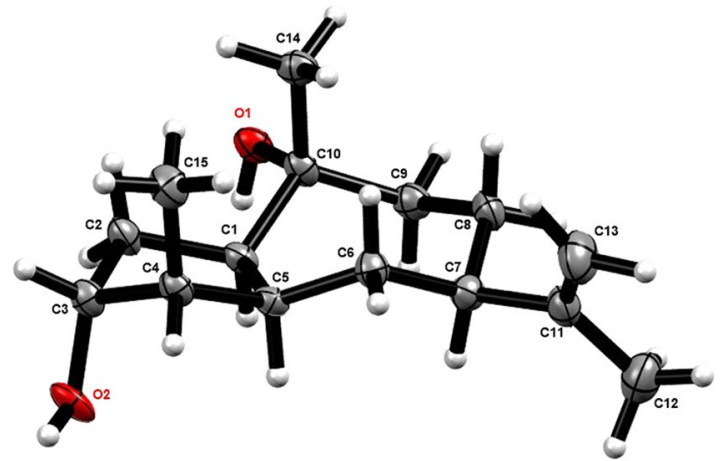

Figure 3. X-ray crystallographic structure of $\mathbf{1}$.

(50 $\mu \mathrm{m}$, Daiso, Japan). TLC precoated silica gel plates (GF254, Qingdao Marine Chemistry Co. Ltd, Qingdao, China) were used for TLC detection. All chemical reagents used were analytical grade.

Fungal Identification and Fermentation. The fungus Graphostroma sp. MCCC 3A00421 was isolated from a hydrothermal sulfide deposit in August 2012 from the Atlantic Ocean (W $13.36^{\circ}, \mathrm{S} 15.17^{\circ}$ ) at a depth of $-2721 \mathrm{~m}$. It was identified to be Graphostroma genus on the basis of comparison of its ITS1-5.8S-ITS2 rRNA gene sequence (KM190888) with those deposited in GenBank of the NCBI using a BLAST searching tool. The 


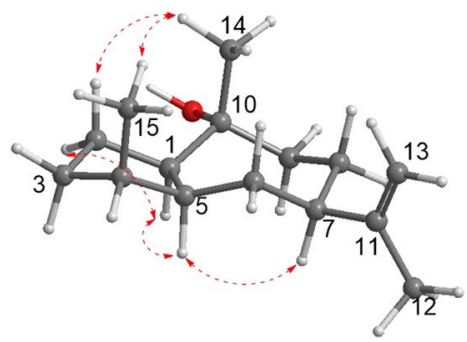

2

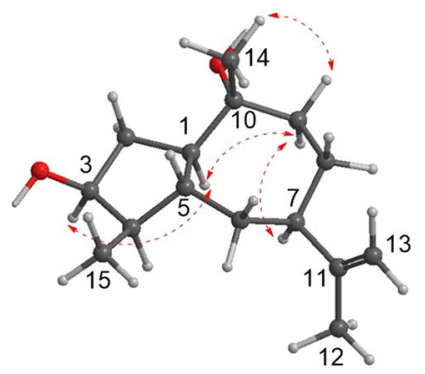

3

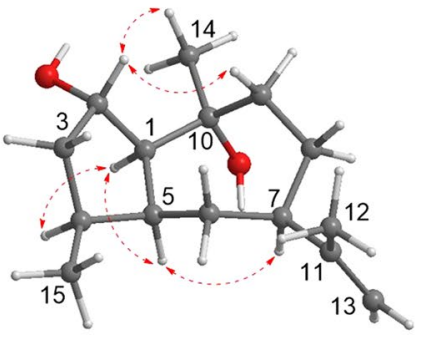

5

Figure 4. Key NOESY correlations of $\mathbf{2 , 3}$, and $\mathbf{5}$.

voucher strain was deposited at the Marine Culture Collection of China (MCCC) with the accession number MCCC 3A00421.

The working strain was cultured on a PDA plate medium under $25^{\circ} \mathrm{C}$ for 3 days. Then the fresh mycelia were inoculated into 30 Erlenmeyer flasks $(1 \mathrm{~L}$ ), each containing $120 \mathrm{~mL}$ distilled water and $80 \mathrm{~g}$ rice, and then statically fermented for 28 days at $25^{\circ} \mathrm{C}$.

Extraction, Isolation, and Purification. After 28 days, the fermented cultures were extracted with EtOAc for three times. The EtOAc solution was evaporated under reduced pressure to get an organic extract, which was then partitioned between $\mathrm{MeOH}$ and petroleum ether (PE). The $\mathrm{MeOH}$ fraction was evaporated to get the defatted extract $(7.0 \mathrm{~g})$, which was separated by column chromatography (CC) over ODS eluting with gradient $\mathrm{MeOH}-\mathrm{H}_{2} \mathrm{O}(5 \rightarrow 100 \%)$ to yield 24 fractions (F1-F24). Fraction F7 (624 mg) was subjected to CC over Sephadex LH-20 (MeOH), followed by repeated CC on silica gel $\left(\mathrm{CHCl}_{3}-\mathrm{MeOH}, 15: 1\right.$; EtOAc- $\mathrm{MeOH}$, 50:1) to yield $10(48.4 \mathrm{mg})$ and $12(10.2 \mathrm{mg})$. Fraction F9 $(420 \mathrm{mg})$ was purified by CC over Sephadex LH-20 $(\mathrm{MeOH})$ to get three subfractions (SF9-1-9-3). SF9-1 was purified by CC over silica gel using PE-acetone (5:1), followed by Prep. TLC $\left(\mathrm{CHCl}_{3}-\mathrm{MeOH}, 8: 1\right)$ to provide $7(12.6 \mathrm{mg})$. SF9-3 was subjected to CC over silica gel $\left(\mathrm{CHCl}_{3}-\mathrm{MeOH}, 15: 1\right)$ to afford $\mathbf{8}(8.2 \mathrm{mg})$. Compounds $\mathbf{6}(18.8 \mathrm{mg})$ and $\mathbf{1 3}(24.2 \mathrm{mg})$ were isolated from fraction F10 (603 mg) by CC over Sephadex LH-20 (MeOH) and silica gel (PE-acetone, 3:1; PE-EtOAc, 3:1 $\rightarrow 1: 1)$. Fraction F14 (389 mg) was subjected to CC over silica gel using gradient PE-EtOAc to give seven subfractions (SF14-1SF14-7). SF14-2 was chromatographed on Sephadex LH-20 (MeOH) to yield 4 (26.0 mg). SF14-3 was purified by Prep. TLC (PE-acetone, 2:1) to get 1 (14.7 mg). Compound $3(10.2 \mathrm{mg})$ was isolated from SF14-5 by Prep. TLC (EtOAc-acetone, 30:1). SF14-6 was separated by CC over Sephadex LH-20 (MeOH) and silica gel $\left(\mathrm{CHCl}_{3}-\mathrm{MeOH}\right.$, 20:1) to yield $11(39.2 \mathrm{mg})$. Fraction F16 (462 mg) was subjected to CC on Sephadex LH-20 (MeOH) and silica gel $\left(\mathrm{CHCl}_{3}-\mathrm{MeOH}, 100: 1 \rightarrow 1: 1\right.$; EtOAc-MeOH, 200:1 $\rightarrow 10: 1$; and PE-acetone, 5:1) to yield 2 (23.0 mg), 5 (15.5 mg), and 9 (11.6 mg).

Graphostromane A (1): colorless oil; $[\alpha]_{\mathrm{D}}^{25}-22.0$ (c 0.58, MeOH); ${ }^{1} \mathrm{H}$ and ${ }^{13} \mathrm{C}$ NMR data, Tables 1 and 2; HRESIMS $m / z 239.2044[\mathrm{M}+\mathrm{H}]^{+}$(calcd for $\mathrm{C}_{15} \mathrm{H}_{27} \mathrm{O}_{2}, 239.2011$ ).

Graphostromane B (2): colorless oil; $[\alpha]_{\mathrm{D}}^{25}-28.5$ (c 2.16, MeOH); ${ }^{1} \mathrm{H}$ and ${ }^{13} \mathrm{C}$ NMR data, Tables 1 and 2; HRESIMS $m / z 223.2057[\mathrm{M}+\mathrm{H}]^{+}$(calcd for $\left.\mathrm{C}_{15} \mathrm{H}_{27} \mathrm{O}, 223.2062\right)$.

Graphostromane $C(3)$ : colorless oil; $[\alpha]_{\mathrm{D}}^{25}-8.5(c 0.25, \mathrm{MeOH}) ;{ }^{1} \mathrm{H}$ and ${ }^{13} \mathrm{C} \mathrm{NMR}$ data, Tables 1 and 2; HRESIMS $m / z 261.1829[\mathrm{M}+\mathrm{Na}]^{+}$(calcd for $\mathrm{C}_{15} \mathrm{H}_{26} \mathrm{O}_{2} \mathrm{Na}, 261.1830$ ).

Graphostromane $\mathrm{D}(4)$ : colorless oil; $[\alpha]_{\mathrm{D}}^{25}+15.4($ c $0.73, \mathrm{MeOH}) ;{ }^{1} \mathrm{H}$ and ${ }^{13} \mathrm{C}$ NMR data, Tables 1 and 2; HRESIMS $m / z 237.1851[\mathrm{M}+\mathrm{H}]^{+}\left(\right.$calcd for $\left.\mathrm{C}_{15} \mathrm{H}_{25} \mathrm{O}_{2}, 237.1855\right)$.

Graphostromane E (5): colorless oil; $[\alpha]_{\mathrm{D}}^{25}+8.13(c 0.39, \mathrm{MeOH}) ;{ }^{1} \mathrm{H}$ and ${ }^{13} \mathrm{C}$ NMR data, Tables 1 and 2; HRESIMS $m / z 261.1833[\mathrm{M}+\mathrm{Na}]^{+}$(calcd for $\mathrm{C}_{15} \mathrm{H}_{26} \mathrm{O}_{2} \mathrm{Na}, 261.1830$ ).

Graphostromane $F(6)$ : colorless oil; $[\alpha]_{\mathrm{D}}^{25}+3.41(c 0.44, \mathrm{MeOH}) ;{ }^{1} \mathrm{H}$ and ${ }^{13} \mathrm{C}$ NMR data, Tables 1 and 2; HRESIMS $m / z 277.1767[\mathrm{M}+\mathrm{Na}]^{+}$(calcd for $\mathrm{C}_{15} \mathrm{H}_{26} \mathrm{O}_{3} \mathrm{Na}, 277.1780$ ).

Graphostromane $G(7)$ : colorless oil; $[\alpha]_{\mathrm{D}}^{25}+6.0(c 0.56, \mathrm{MeOH}) ;{ }^{1} \mathrm{H}$ and ${ }^{13} \mathrm{C}$ NMR data, Tables 1 and 2; HRESIMS $m / z 337.1979[\mathrm{M}+\mathrm{Na}]^{+}\left(\right.$calcd for $\left.\mathrm{C}_{17} \mathrm{H}_{30} \mathrm{O}_{5} \mathrm{Na}, 337.1991\right)$.

Graphostromane $H(\boldsymbol{8})$ : colorless oil; $[\alpha]_{\mathrm{D}}^{25}-21.0($ c $0.25, \mathrm{MeOH}) ;{ }^{1} \mathrm{H}$ and ${ }^{13} \mathrm{C}$ NMR data, Tables 1 and 2; HRESIMS $m / z 295.1878[\mathrm{M}+\mathrm{Na}]^{+}$(calcd for $\mathrm{C}_{15} \mathrm{H}_{28} \mathrm{O}_{4} \mathrm{Na}, 295.1885$ ).

Graphostromane I (9): colorless oil; $[\alpha]_{\mathrm{D}}^{25}-10.3($ c $0.12, \mathrm{MeOH}) ;{ }^{1} \mathrm{H}$ and ${ }^{13} \mathrm{C}$ NMR data, Tables 1 and 2; HRESIMS $m / z 277.1783[\mathrm{M}+\mathrm{Na}]^{+}$(calcd for $\mathrm{C}_{15} \mathrm{H}_{26} \mathrm{O}_{3} \mathrm{Na}, 277.1780$ ).

Compound 10: ${ }^{13} \mathrm{C}$ NMR $\left(\mathrm{CD}_{3} \mathrm{OD}, 100 \mathrm{MHz}\right) \delta_{\mathrm{C}} 63.4(\mathrm{CH}-1), 75.1(\mathrm{CH}-2), 43.3\left(\mathrm{CH}_{2}-3\right), 37.3(\mathrm{CH}-4), 47.3$ (CH-5), $25.8\left(\mathrm{CH}_{2}-6\right), 45.7(\mathrm{CH}-7), 26.4\left(\mathrm{CH}_{2}-8\right), 39.5\left(\mathrm{CH}_{2}-9\right), 75.4(\mathrm{C}-10), 76.6(\mathrm{C}-11), 69.2\left(\mathrm{CH}_{2}-12\right), 19.0$ (Me-13), $29.6(\mathrm{Me}-14), 16.7$ (Me-15).

Preparation of $(R)$ - and (S)-MPA Esters of Compounds 3, 5, and 7. Compound 3 (2.0 mg) was dissolved in $\mathrm{CHCl}_{3}(600 \mu \mathrm{L})$. Then $(R)$-MPA $(2.5 \mathrm{mg})$, DCC $(2.5 \mathrm{mg})$, and DMAP (2.5 mg) were added. After stirred $16 \mathrm{~h}$ at room temperature, the reactive products were subjected to CC over silica gel (PE-acetone, 3:1) to give the $R$-MPA ester 3a (1.7 mg). Similarly, the $S$-MPA ester $\mathbf{3 b}(1.9 \mathrm{mg})$ was obtained from $(S)$-MPA. Analogue treatment of compounds 5 and 7 separately with $(R)$-MPA and $(S)$-MPA obtained $(R)$-MPA esters (5a and 7a) and $(S)$-MPA esters ( $\mathbf{5 b}$ and $\mathbf{7 b})$, respectively. 

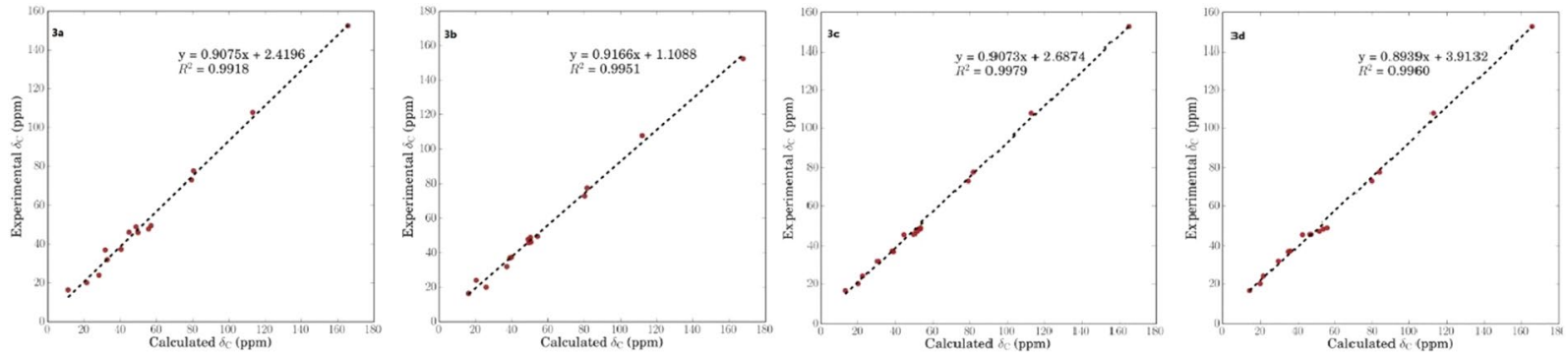

Figure 5. Calculated ${ }^{13} \mathrm{C}$ NMR spectroscopic data of four possible stereoisomers of $\mathbf{3}$ (3a, 3b, 3c, and $\mathbf{3 d}$ ) at mPW1PW91/6-311+G(2d,p) level in pyridine- $d_{5}$.

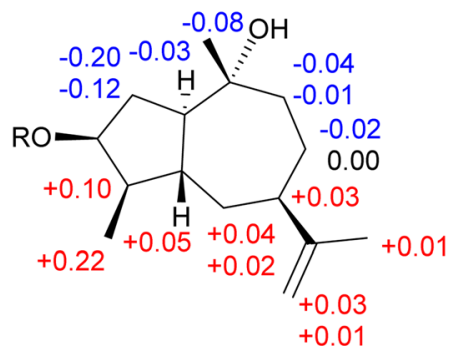

3a $\mathrm{R}=(R)-\mathrm{MPA}$

3b $\mathrm{R}=(S)-\mathrm{MPA}$

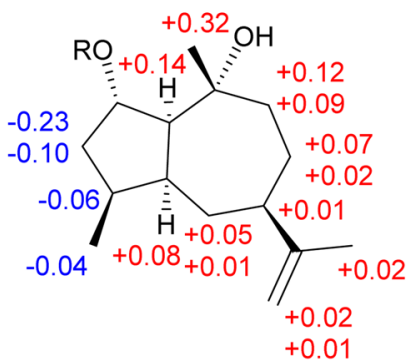

$5 \mathrm{a} \mathrm{R}=(R)-\mathrm{MPA}$ 5b R $=(S)-M P A$

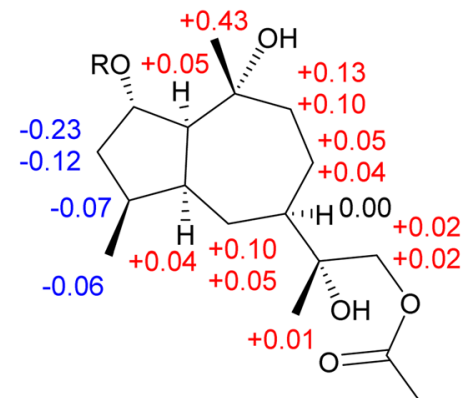

7a $\mathrm{R}=(R)-\mathrm{MPA}$ 7b $\mathrm{R}=(S)-\mathrm{MPA}$

Figure 6. $\Delta \delta_{\mathrm{H}}\left(\delta_{\mathrm{H}}{ }^{R}-\delta_{\mathrm{H}}^{S}\right)$ values of $(R)$ - and $(S)$-MPA esters of $\mathbf{3}, \mathbf{5}$, and 7 in $\mathrm{CDCl}_{3}$.

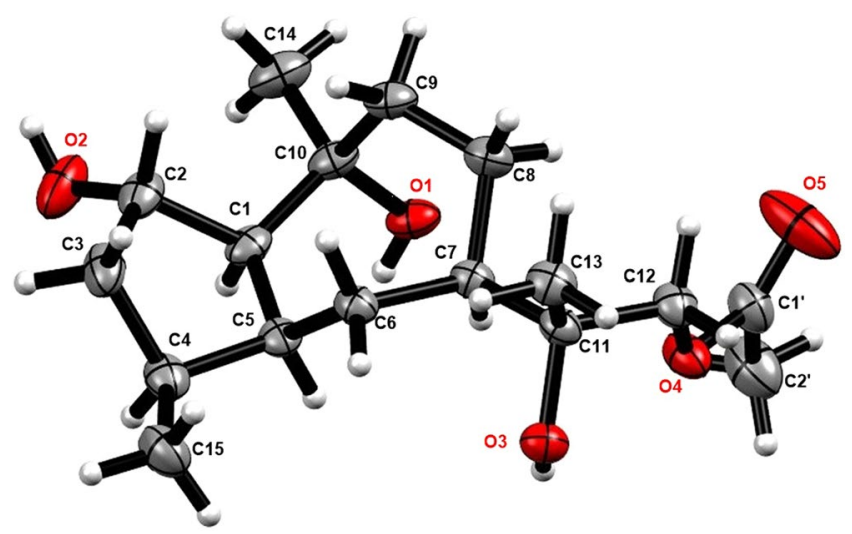

Figure 7. X-ray crystallographic structure of 7.

(R)-MPA ester of $3(3 a):{ }^{1} \mathrm{H} \mathrm{NMR}\left(\mathrm{CDCl}_{3}, 400 \mathrm{MHz}\right) \delta_{\mathrm{H}} 7.32-7.47(5 \mathrm{H}, \mathrm{m}$, phenyl protons $), 4.78(1 \mathrm{H}, \mathrm{s}, \mathrm{CH}$ of MPA), $3.43(3 \mathrm{H}, \mathrm{s}$, OMe of MPA), $2.25(1 \mathrm{H}, \mathrm{m}, \mathrm{H}-1), 1.44(1 \mathrm{H}, \mathrm{m}, \mathrm{H}-2 \mathrm{a}), 2.22(1 \mathrm{H}, \mathrm{m}, \mathrm{H}-2 \mathrm{~b}), 4.68(1 \mathrm{H}, \mathrm{m}, \mathrm{H}-3)$, $1.61(1 \mathrm{H}, \mathrm{m}, \mathrm{H}-4), 1.66(1 \mathrm{H}, \mathrm{m}, \mathrm{H}-5), 1.36(1 \mathrm{H}, \mathrm{m}, \mathrm{H}-6 \mathrm{a}), 1.69(1 \mathrm{H}, \mathrm{m}, \mathrm{H}-6 \mathrm{~b}), 1.96(1 \mathrm{H}, \mathrm{dt}, J=2.2,11.5 \mathrm{~Hz}$, H-7), $1.46(1 \mathrm{H}, \mathrm{m}, \mathrm{H}-8 \mathrm{a}), 1.71(1 \mathrm{H}, \mathrm{m}, \mathrm{H}-8 \mathrm{~b}), 1.63(1 \mathrm{H}, \mathrm{m}, \mathrm{H}-9 \mathrm{a}), 1.85$ (1 H, ddd, J=2.6, 4.7, 8.1 Hz, H-9b), $1.70(3 \mathrm{H}, \mathrm{s}, \mathrm{Me}-12), 4.62(1 \mathrm{H}, \mathrm{br} \mathrm{s}, \mathrm{H}-13 \mathrm{a}), 4.65(1 \mathrm{H}, \mathrm{br} \mathrm{s}, \mathrm{H}-13 \mathrm{~b}), 1.18(3 \mathrm{H}, \mathrm{s}, \mathrm{Me}-14), 0.91(3 \mathrm{H}, \mathrm{d}, J=6.2 \mathrm{~Hz}$, Me-15).

(S)-MPA ester of $3(3 \boldsymbol{b}):{ }^{1} \mathrm{H} \mathrm{NMR}\left(\mathrm{CDCl}_{3}, 400 \mathrm{MHz}\right) \delta_{\mathrm{H}} 7.33-7.47(5 \mathrm{H}, \mathrm{m}$, phenyl protons), $4.77(1 \mathrm{H}, \mathrm{s}, \mathrm{CH}$ of MPA), $3.44(3 \mathrm{H}, \mathrm{s}$, OMe of MPA), $2.28(1 \mathrm{H}, \mathrm{m}, \mathrm{H}-1), 1.64(1 \mathrm{H}, \mathrm{m}, \mathrm{H}-2 \mathrm{a}), 2.34(1 \mathrm{H}, \mathrm{m}, \mathrm{H}-2 \mathrm{~b}), 4.63(1 \mathrm{H}, \mathrm{m}, \mathrm{H}-3)$, $1.51(1 \mathrm{H}, \mathrm{m}, \mathrm{H}-4), 1.61(1 \mathrm{H}, \mathrm{m}, \mathrm{H}-5), 1.34(1 \mathrm{H}, \mathrm{m}, \mathrm{H}-6 \mathrm{a}), 1.65(1 \mathrm{H}, \mathrm{m}, \mathrm{H}-6 \mathrm{~b}), 1.93(1 \mathrm{H}, \mathrm{t}, J=10.8 \mathrm{~Hz}, \mathrm{H}-7)$, $1.48(1 \mathrm{H}, \mathrm{m}, \mathrm{H}-8 \mathrm{a}), 1.71(1 \mathrm{H}, \mathrm{m}, \mathrm{H}-8 \mathrm{~b}), 1.64(1 \mathrm{H}, \mathrm{m}, \mathrm{H}-9 \mathrm{a}), 1.89(1 \mathrm{H}, \mathrm{m}, \mathrm{H}-9 \mathrm{~b}), 1.69(3 \mathrm{H}, \mathrm{s}, \mathrm{Me}-12), 4.61(1 \mathrm{H}$, br s, H-13a), 4.64 (1 H, br s, H-13b), $1.26(3 \mathrm{H}, \mathrm{s}, \mathrm{Me}-14), 0.69(3 \mathrm{H}, \mathrm{d}, J=6.5 \mathrm{~Hz}, \mathrm{Me}-15)$.

(R)-MPA ester of $5(5 a):{ }^{1} \mathrm{H} \mathrm{NMR}\left(\mathrm{CDCl}_{3}, 400 \mathrm{MHz}\right) \delta_{\mathrm{H}} 7.32-7.47(5 \mathrm{H}, \mathrm{m}$, phenyl protons $), 4.78(1 \mathrm{H}, \mathrm{s}, \mathrm{CH}$ of MPA), 3.43 (3 H, s, OMe of MPA), $2.35(1 \mathrm{H}, \mathrm{m}, \mathrm{H}-1), 5.25(1 \mathrm{H}, \mathrm{m}, \mathrm{H}-2), 1.51(1 \mathrm{H}, \mathrm{m}, \mathrm{H}-3 \mathrm{a}), 1.64(1 \mathrm{H}, \mathrm{m}, \mathrm{H}-3 \mathrm{~b})$, $2.16(1 \mathrm{H}, \mathrm{m}, \mathrm{H}-4), 2.30(1 \mathrm{H}, \mathrm{m}, \mathrm{H}-5), 1.23(1 \mathrm{H}, \mathrm{m}, \mathrm{H}-6 \mathrm{a}), 1.45(1 \mathrm{H}, \mathrm{m}, \mathrm{H}-6 \mathrm{~b}), 2.11(1 \mathrm{H}, \mathrm{m}, \mathrm{H}-7), 1.45(1 \mathrm{H}, \mathrm{m}$, 

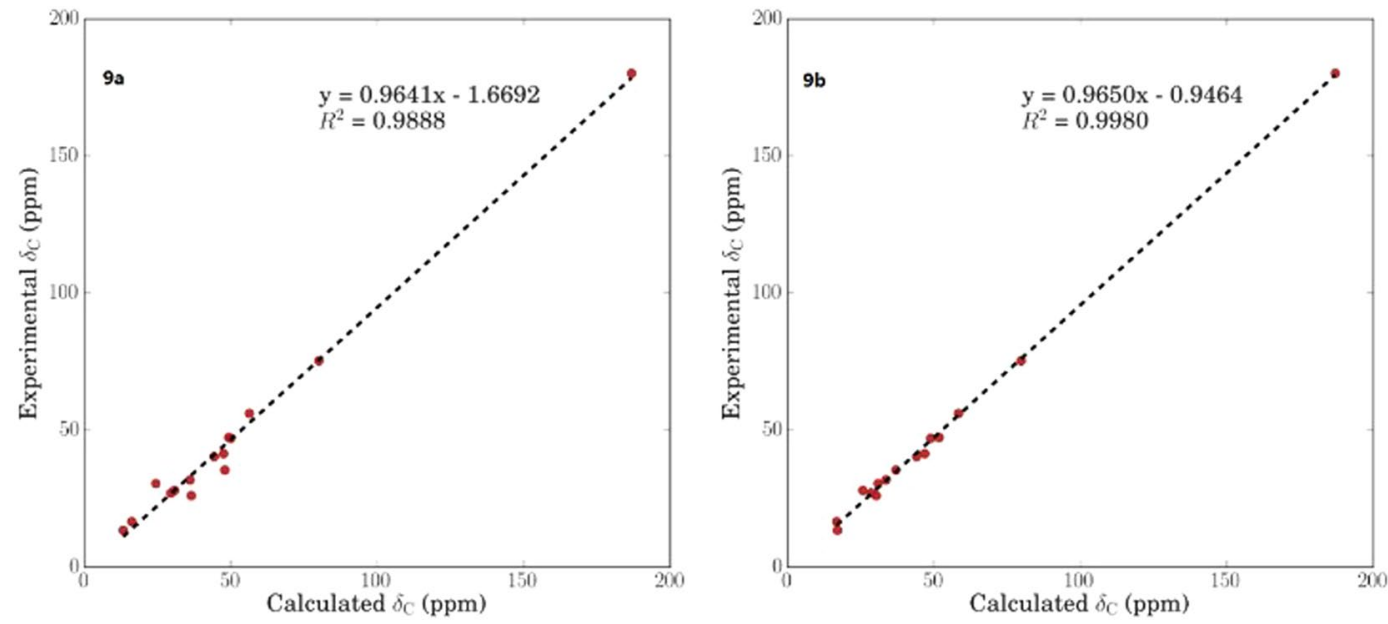

Figure 8. Calculated ${ }^{13} \mathrm{C}$ NMR spectroscopic data of a pair of C-11 epimers of $(1 R, 4 S, 5 S, 7 R, 10 R, 11 S)-9 \mathbf{a}$ and $(1 R, 4 S, 5 S, 7 R, 10 R, 11 R)-9 \mathbf{b}$ at mPW1PW91/6-311 + G(2d,p) level in $\mathrm{CD}_{3} \mathrm{OD}$.

\begin{tabular}{|l|c|l|}
\hline Compounds & $\mathrm{IC}_{\mathbf{5 0}}(\boldsymbol{\mu} \mathbf{M})$ & $\mathrm{CC}_{\mathbf{5 0}}(\boldsymbol{\mu M})$ \\
\hline $\mathbf{1}$ & 310.1 & $>50$ \\
\hline $\mathbf{2}$ & 103.2 & $>50$ \\
\hline $\mathbf{3}$ & 112.6 & $>50$ \\
\hline $\mathbf{4}$ & 72.9 & $>50$ \\
\hline $\mathbf{5}$ & 138.2 & $>50$ \\
\hline $\mathbf{6}$ & 14.2 & $>50$ \\
\hline $\mathbf{7}$ & 101.0 & $>50$ \\
\hline $\mathbf{8}$ & 165.8 & $>50$ \\
\hline $\mathbf{9}$ & 79.1 & $>50$ \\
\hline $\mathbf{1 0}$ & 150.4 & $>50$ \\
\hline $\mathbf{1 1}$ & 141.0 & $>50$ \\
\hline $\mathbf{1 2}$ & 122.4 & $>50$ \\
\hline $\mathbf{1 3}$ & 88.2 & $>50$ \\
\hline Aminoguanidine & & \\
\hline
\end{tabular}

Table 3. Anti-inflammatory activities of compounds 1-13 against LPS-stimulated NO production by in RAW

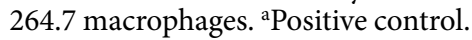

H-8a), 1.75 (1 H, m, H-8b), 1.67 (1 H, m, H-9a), 1.82 (1 H, m, H-9b), 1.69 (3 H, s, Me-12), 4.61 (1 H, br s, H-13a), $4.65(1 \mathrm{H}$, br s, H-13b), $1.22(3 \mathrm{H}, \mathrm{s}, \mathrm{Me}-14), 0.87(3 \mathrm{H}, \mathrm{d}, J=7.0 \mathrm{~Hz}, \mathrm{Me}-15)$.

$(S)-M P A$ ester of $5(5 \boldsymbol{b}):{ }^{1} \mathrm{H}$ NMR $\left(\mathrm{CDCl}_{3}, 400 \mathrm{MHz}\right) \delta_{\mathrm{H}} 7.31-7.44(5 \mathrm{H}, \mathrm{m}$, phenyl protons $), 4.73(1 \mathrm{H}, \mathrm{s}, \mathrm{CH}$ of MPA), $3.41(3 \mathrm{H}, \mathrm{s}, \mathrm{OMe}$ of MPA), $2.21(1 \mathrm{H}, \mathrm{m}, \mathrm{H}-1), 5.15(1 \mathrm{H}, \mathrm{m}, \mathrm{H}-2), 1.74(2 \mathrm{H}, \mathrm{m}, \mathrm{H}-3), 2.22(2 \mathrm{H}, \mathrm{m}, \mathrm{H}-4$ and $\mathrm{H}-5), 1.17(1 \mathrm{H}, \mathrm{m}, \mathrm{H}-6 \mathrm{a}), 1.44(1 \mathrm{H}, \mathrm{m}, \mathrm{H}-6 \mathrm{~b}), 2.10(1 \mathrm{H}, \mathrm{dt}, J=4.1,11.4 \mathrm{~Hz}, \mathrm{H}-7), 1.38(1 \mathrm{H}, \mathrm{m}, \mathrm{H}-8 \mathrm{a}), 1.73$ $(2 \mathrm{H}, \mathrm{m}, \mathrm{H}-8 \mathrm{~b}$ and H-9b), $1.55(1 \mathrm{H}, \mathrm{t}, J=10.1 \mathrm{~Hz}, \mathrm{H}-9 \mathrm{a}), 1.67(3 \mathrm{H}, \mathrm{s}, \mathrm{Me}-12), 4.60(1 \mathrm{H}, \mathrm{br} \mathrm{s}, \mathrm{H}-13 \mathrm{a}), 4.63(1 \mathrm{H}$, br s, H-13b), 0.90 (3 H, s, Me-14), $0.91(3 \mathrm{H}, \mathrm{d}, J=7.0 \mathrm{~Hz}, \mathrm{Me}-15)$.

(R)-MPA ester of $7(7 \boldsymbol{a}):{ }^{1} \mathrm{H}$ NMR $\left(\mathrm{CDCl}_{3}, 400 \mathrm{MHz}\right) \delta_{\mathrm{H}} 7.32-7.44(5 \mathrm{H}, \mathrm{m}$, phenyl protons $), 4.74(1 \mathrm{H}, \mathrm{s}, \mathrm{CH}$ of MPA), $3.41(3 \mathrm{H}, \mathrm{s}, \mathrm{OMe}$ of MPA), $2.31(1 \mathrm{H}, \mathrm{t}, J=6.9 \mathrm{~Hz}, \mathrm{H}-1), 5.17(1 \mathrm{H}, \mathrm{dt}, J=2.9,7.6 \mathrm{~Hz}, \mathrm{H}-2), 1.45(1 \mathrm{H}, \mathrm{m}$, H-3a), 1.68 (1 H, m, H-3b), 2.16 (1 H, m, H-4), 2.18 (1 H, m, H-5), 0.91 (1 H, m, H-6a), 1.81 (1 H, m, H-6b), $1.77(1 \mathrm{H}$, m, H-7), 1.19 (1 H, m, H-8a), $1.76(1 \mathrm{H}, \mathrm{m}, \mathrm{H}-8 \mathrm{~b}), 1.59$ (1 H, m, H-9a), $1.82(1 \mathrm{H}, \mathrm{m}, \mathrm{H}-9 \mathrm{~b}), 1.06$ (3 H, s, Me-12), 3.97 $(1 \mathrm{H}, \mathrm{d}, J=11.5 \mathrm{~Hz}, \mathrm{H}-13 \mathrm{a}), 4.06(1 \mathrm{H}, \mathrm{d}, J=11.5 \mathrm{~Hz}, \mathrm{H}-13 \mathrm{~b}), 1.14(3 \mathrm{H}, \mathrm{s}, \mathrm{Me}-14), 0.90(3 \mathrm{H}, \mathrm{d}, J=6.5 \mathrm{~Hz}, \mathrm{Me}-15)$.

$(S)$-MPA ester of $7(7 \boldsymbol{b}):{ }^{1} \mathrm{H} \mathrm{NMR}\left(\mathrm{CDCl}_{3}, 400 \mathrm{MHz}\right) \delta_{\mathrm{H}} 7.32-7.42(5 \mathrm{H}, \mathrm{m}$, phenyl protons $), 4.71(1 \mathrm{H}, \mathrm{s}, \mathrm{CH}$ of MPA), 3.40 (3 H, s, OMe of MPA), $2.16(1 \mathrm{H}, \mathrm{m}, \mathrm{H}-1), 5.05(1 \mathrm{H}, \mathrm{dt}, J=2.8,7.7 \mathrm{~Hz}, \mathrm{H}-2), 1.68(1 \mathrm{H}, \mathrm{m}, \mathrm{H}-3 \mathrm{a})$, $1.80(1 \mathrm{H}, \mathrm{m}, \mathrm{H}-3 \mathrm{~b}), 2.23(1 \mathrm{H}, \mathrm{m}, \mathrm{H}-4), 2.21(1 \mathrm{H}, \mathrm{m}, \mathrm{H}-5), 0.86(1 \mathrm{H}, \mathrm{m}, \mathrm{H}-6 \mathrm{a}), 1.71(2 \mathrm{H}, \mathrm{m}, \mathrm{H}-6 \mathrm{~b}$ and H-8b), $1.77(1 \mathrm{H}, \mathrm{m}, \mathrm{H}-7), 1.15(1 \mathrm{H}, \mathrm{m}, \mathrm{H}-8 \mathrm{a}), 1.46(1 \mathrm{H}, \mathrm{m}, \mathrm{H}-9 \mathrm{a}), 1.72(1 \mathrm{H}, \mathrm{m}, \mathrm{H}-9 \mathrm{~b}), 1.05(3 \mathrm{H}, \mathrm{s}, \mathrm{Me}-12), 3.95(1 \mathrm{H}, \mathrm{d}$, $J=11.5 \mathrm{~Hz}, \mathrm{H}-13 \mathrm{a}), 4.04(1 \mathrm{H}, \mathrm{d}, J=11.5 \mathrm{~Hz}, \mathrm{H}-13 \mathrm{~b}), 0.71(3 \mathrm{H}, \mathrm{s}, \mathrm{Me}-14), 0.96(3 \mathrm{H}, \mathrm{d}, J=6.8 \mathrm{~Hz}, \mathrm{Me}-15)$.

X-ray Crystal Data of Compounds 1 and 7. Graphostromane A (1) was obtained as colorless crystals. The monoclinic crystals $\left(0.1 \times 0.2 \times 0.8 \mathrm{~mm}^{3}\right)$ was recorded on a Bruker D8 Advance X-ray single-crystal diffractometer with $\mathrm{Cu} \mathrm{K \alpha}$ radiation. Crystal data of 1 : empirical formula $\mathrm{C}_{15} \mathrm{H}_{26} \mathrm{O}_{2}, \mathrm{M}=236.36$; space group $\mathrm{P} 2{ }_{1}$, unit cell dimensions $\mathrm{a}=6.3808$ (4) $\AA, \mathrm{b}=7.7943$ (4) $\AA, \mathrm{c}=13.9771$ (8) $\AA, \alpha=90.00^{\circ}, \beta=101.329(6)^{\circ}$, 
$\gamma=90.00^{\circ}, \mathrm{V}=681.59(7) \AA^{3}, \mathrm{Z}=2, \mathrm{D}_{\text {calcd }}=1.1516 \mathrm{~g} / \mathrm{cm}^{3}, \mu=0.579 \mathrm{~mm}^{-1}, \mathrm{~F}(000)=260.8$; A total of 6871 reflections were collected in the range of $6.44^{\circ}<2 \theta<124.16^{\circ}$, of which 2128 independent reflections $\left[\mathrm{R}_{\text {int }}=0.0548\right.$, $\left.\mathrm{R}_{\text {sigma }}=0.0552\right]$ were used for the analysis. The structure was solved by the direct methods with the SHELXL-97 program and refined using full-matrix least-squares difference Fourier techniques. The final R indexes [all data] gave $\mathrm{R}_{1}=0.0642, \mathrm{wR}_{2}=0.1712$ and the Flack parameter $=-0.1$ (3). Crystallographic data of 1 have been deposited in the Cambridge Crystallographic Data Center (deposition number CCDC 1529237).

Colorless crystals of 7 were obtained from $\mathrm{MeOH}$. The monoclinic crystals $\left(0.3 \times 0.4 \times 0.8 \mathrm{~mm}^{3}\right)$ was measured on a Bruker D8 Advance X-ray single-crystal diffractometer with $\mathrm{Cu} \mathrm{K} \alpha$ radiation. Crystal data of 7: empirical formula $\mathrm{C}_{17} \mathrm{H}_{30} \mathrm{O}_{5}, \mathrm{M}=314.42$; space group $\mathrm{C} 2$, unit cell dimensions $\mathrm{a}=12.2072(4) \AA$, $\mathrm{b}=14.3161$ (3) $\AA, c=13.4457$ (4) $\AA, \alpha=\gamma=90.00^{\circ}, \beta=114.561(4)^{\circ}$, Volume $=2137.16(12) \AA^{3}, Z=9, D_{\text {calcd }}=1.173 \mathrm{~g} / \mathrm{cm}^{3}$, $\mu=0.774 \mathrm{~mm}^{-1}, \mathrm{~F}(000)=828.0$; A total of 11239 reflections were collected in the range of $7.228^{\circ}<2 \theta<123.85^{\circ}$, of which 3337 independent reflections $\left[R_{\text {int }}=0.0330, R_{\text {sigma }}=0.0280\right]$ were used for the analysis. The final $R$ indexes [all data] gave $\mathrm{R}_{1}=0.0368, \mathrm{wR}_{2}=0.1006$ and the Flack parameter $=0.04$ (7). Crystallographic data of 7 have been deposited in the Cambridge Crystallographic Data Center (deposition number CCDC 1577811).

Theoretical Calculation of CMR Data. The calculated CMR data of $\mathbf{3}$ and $\mathbf{9}$ were carried out by Gaussian $09^{23}$. Conformational analyses were initially performed using Confab ${ }^{24}$ with MMFF94 force field for configurations of both compounds. The conformers, which were chosen for CMR calculations with Boltzmann-population over 1\%, were firstly optimized at PM6 by semi-empirical theory method to filter some conformers with low Boltzmann-populations. Then, the remaining conformers were optimized at B3LYP/6-31 $+\mathrm{G}(\mathrm{d}, \mathrm{p})$ in gas phase. The CMR calculation was conducted by the Gauge-Including Atomic Orbitals (GIAO) method at mPW1PW91/6-311 + G(2d,p) level using the IEFPCM model in pyridine for 3, whereas in $\mathrm{MeOH}$ for 9, respectively. Finally, the TMS-corrected ${ }^{13} \mathrm{C}$ NMR chemical shift values were averaged according to Boltzmann distribution for each conformer and fitting to the experimental values by linear regression. The calculated CMR chemical shift values of TMS in pyridine and in $\mathrm{MeOH}$ were $187.3194 \mathrm{ppm}$ and $187.3772 \mathrm{ppm}$, respectively.

Anti-Inflammatory Assay. This experiment was conducted according the reported procedure ${ }^{25}$.

\section{References}

1. Chen, Z. et al. Rupestonic acids B-G, NO inhibitory sesquiterpenoids from Artemisia rupestris. Bioorg. Med. Chem. Lett. 24, 4318-4322 (2014).

2. Yang, Y. J. et al. Sesquiterpenoids and tirucallane triterpenoids from the roots of Scorzonera divaricata. Phytochemistry 124, 86-98 (2016).

3. Yang, M. C., Choi, S. U., Choi, W. S., Kim, S. Y. \& Lee, K. R. Guaiane sesquiterpene lactones and amino acid-sesquiterpene lactone conjugates from the aerial parts of Saussurea pulchella. J. Nat. Prod. 71, 678-683 (2008).

4. Ma, Cm, Nakamura, N., Hattori, M., Zhu, S. \& Komatsu, K. Guaiane dimers and germacranolide from Artemisia caruifolia. J. Nat. Prod. 63, 1626-1629 (2000).

5. Zhang, Y. L. et al. Xylopiana A, a dimeric guaiane with a case-shaped core from Xylopia vielana: structural elucidation and biomimetic conversion. Org. Lett. 19, 3013-3016 (2017).

6. Takaya, Y. et al. Novel antimalarial guaiane-type sesquiterpenoids from Nardostachys chinensis roots. Tetrahedron Lett. 39, 1361-1364 (1998).

7. Takaya, Y. et al. Novel guaiane endoperoxides, nardoguaianone A-D, from Nardostachys chinensis roots and their antinociceptive and antimalarial activities. Tetrahedron 56, 7673-7678 (2000).

8. Yang, Y. et al. Anti-emetic principles of Pogostemon cablin (Blanco) Benth. Phytomedicine. 6, 89-93 (1999).

9. Ratnayake, R., Covell, D., Ransom, T. T., Gustafson, K. R. \& Beutler, J. A. Englerin A, a selective inhibitor of renal cancer cell growth, from Phyllanthus engleri. Org. Lett. 11, 57-60 (2009).

10. Wang, S. et al. Sesquiterpenes from Artemisia argyi: absolute configurations and biological activities. Eur. J. Org. Chem. 2014, 973-983 (2014).

11. Hyldgaard, M. G. et al. Guaianolides and a seco-eudesmane from the resinous exudates of cushion bush (Leucophyta brownii) and evaluation of their cytostatic and anti-inflammatory activity. J. Nat. Prod. 78, 1877-1885 (2015).

12. Liu, Y. et al. Guaiane-type sesquiterpenes from Curcuma phaeocaulis and their inhibitory effects on nitric oxide production. J. Nat. Prod. 76, 1150-1156 (2013).

13. Li, H. M. et al. Guaiane-type sesquiterpenoids from Alismatis rhizoma and their anti-inflammatory activity. Chem. Pharm. Bull. 65, 403-407 (2017).

14. Chakraborty, K., Lipton, A. P., Paulraj, R. \& Chakraborty, R. D. Guaiane sesquiterpenes from seaweed Ulva fasciata Delile and their antibacterial properties. Eur. J. Med. Chem. 45, 2237-2244 (2010).

15. Wu, S. H. et al. Guaiane sesquiterpenes and isopimarane diterpenes from an endophytic fungus Xylaria sp. Phytochemistry 105, 197-204 (2014).

16. Fraga, B. M. Natural sesquiterpenoids. Nat. Prod. Rep. 30, 1226-1264 (2013).

17. Diep, C. N. et al. Structures and absolute stereochemistry of guaiane sesquiterpenoids from the gorgonian Menella woodin. Tetrahedron Lett. 56, 7001-7004 (2015).

18. Amand, S. et al. Guaiane sesquiterpenes from Biscogniauxia nummularia featuring potent antigerminative activity. J. Nat. Prod. 75 , 798-801 (2012).

19. Wei, H. et al. Sesquiterpenes and other constituents of Xylaria sp. NC1214, a fungal endophyte of the moss Hypnum sp. Phytochemistry 118, 102-108 (2015).

20. Niu, S. et al. Sesquiterpenes from a deep-sea-derived fungus Graphostroma sp. MCCC 3A00421. Tetrahedron 73, 7267-7273 (2017).

21. Freire, F. et al. Relative and absolute stereochemistry of secondary/secondary diols: Low-temperature ${ }^{1} \mathrm{H}$ NMR of their bis-MPA esters. J. Org. Chem. 72, 2297-2301 (2007).

22. Huang, R., Xie, X. S., Fang, X. W., Ma, K. X. \& Wu, S. H. Five new guaiane sesquiterpenes from the endophytic fungus Xylaria sp. YM 311647 of Azadirachta indica. Chem. Biodivers. 12, 1281-1286 (2015).

23. Gaussian 09 (Revision D.01) (Gaussian, Inc., Wallingford, CT, 2009).

24. O'Boyle, N. M., Vandermeersch, T. \& Hutchison, G. R. Confab-generation of diverse low energy conformers. J. Cheminform. 3, 3-8 (2011).

25. Yang, X. W. et al. Anti-inflammatory and anti-tumour effects of Abies georgei extracts. J. Pharm. Pharmacol. 60, 937-941 (2008). 


\section{Acknowledgements}

This work was supported by grants from Xiamen Ocean Economic Innovation and Development Demonstration Project (16PZP001SF16), the National Natural Science Foundation of China (41676130 and 21372233), and the China Postdoctoral Science Foundation (2016M602056).

\section{Author Contributions}

Xian-Wen Yang designed the project; Siwen Niu isolated compounds and determined their structures; Chun-Lan Xie and Jin-Mei Xia conducted the anti-inflammatory experiments; Zhu-Hua Luo and Zongze Shao isolated and identified the fungus; Siwen Niu and Xian-Wen Yang wrote and revised the paper. All authors reviewed the manuscript.

\section{Additional Information}

Supplementary information accompanies this paper at https://doi.org/10.1038/s41598-017-18841-6.

Competing Interests: The authors declare that they have no competing interests.

Publisher's note: Springer Nature remains neutral with regard to jurisdictional claims in published maps and institutional affiliations.

(c) (1) Open Access This article is licensed under a Creative Commons Attribution 4.0 International License, which permits use, sharing, adaptation, distribution and reproduction in any medium or format, as long as you give appropriate credit to the original author(s) and the source, provide a link to the Creative Commons license, and indicate if changes were made. The images or other third party material in this article are included in the article's Creative Commons license, unless indicated otherwise in a credit line to the material. If material is not included in the article's Creative Commons license and your intended use is not permitted by statutory regulation or exceeds the permitted use, you will need to obtain permission directly from the copyright holder. To view a copy of this license, visit http://creativecommons.org/licenses/by/4.0/.

(C) The Author(s) 2018 